\title{
Scalp Psoriasis and Biologic Agents: A Review
}

\author{
Ilias Papadimitriou ${ }^{a}$ Katerina Bakirtzi ${ }^{a} \quad$ Alexander Katoulis $^{b}$ \\ Dimitrios loannides ${ }^{a}$
}

aFirst Dermatology and Venereology Department, School of Medicine Faculty of Health Sciences, Aristotle University, Thessaloniki, Greece; 'becond Department of Dermatology and Venereology, National and Kapodistrian University of Athens Medical School, "Attikon" University Hospital, Athens, Greece

\section{Keywords}

Scalp psoriasis · Review · Biologics · Small molecules · Psoriasis

\begin{abstract}
Scalp-localized psoriasis is common among patients affected with plaque psoriasis, rendering its treatment exceedingly difficult. Furthermore, the symptoms caused by the disease like scaling, erythema, and pruritus, among others, pose a major psychological impact and a significant regression in the quality of life of the affected patients.

Biologics have proved their efficacy in assuaging the symptoms, in terms of Psoriasis Area and Severity Index (PASI) reduction, and offering optimum quality of life, by decreasing the Dermatology Life Quality Index (DLQI) in the patients suffering from plaque psoriasis. Herein, we sought to evaluate the efficacy of biologics and small molecules in controlling the symptoms and their ability to offer long-term maintenance in the disease activity.

(C) 2021 S. Karger AG, Basel
\end{abstract}

\section{Introduction}

Psoriasis is a chronic systemic inflammatory disease that can affect any part of the body. The scalp is one of the most common sites of involvement. Almost $80 \%$ of pa-

karger@karger.com

(C) 2021 S. Karger AG, Basel

www.karger.com/sad

Karger! tients affected with plaque psoriasis eventually develop lesions on the scalp, while in the vast majority of them scalp psoriasis represents the first manifestation of the disease [1-3]. The scalp is one of the anatomic sites, which still constitutes a challenge for the physician, in terms of achieving satisfactory clinical response and meeting patients' needs, despite advances made in the field of biologic and conventional treatments in recent years.

Scalp psoriasis is a major issue to be researched, as it is not only a physical burden, but at the same time inflicts significant psychological stress on the patient, disproportional to the body area affected [4]. Moreover, some authors have suggested that the presence of scalp psoriasis lesions is a possible prognostic marker for the future development of psoriatic arthritis $[5,6]$. Among the symptoms presented in the affected patients, persistent pruritus and intense scaling usually significantly impair the quality of life of the psoriatic patients [2,3].

A recent review article studied the potential mechanisms contributing to the scalp psoriasis phenotype and itch [7]. The mastocytes present in the dermal sheath of the follicles may play a central role in the development of the psoriatic scalp itch. The authors proposed these cells are influenced by the dense scalp innervation, rich skin vasculature, the presence of a large number of sensory, and neuro-mediator skin receptors, alongside the unique hair follicle keratinocyte cycle. Although the number of mastocytes appears increased in the psoriatic lesions, histamine does not seem to 
contribute to the appearance of pruritus. The above conclusion was demonstrated in another study, the result of which showed that the histamine levels were almost identical between patients with psoriasis who presented pruritus and a control group of patients that did not suffer from this symptom, even though the severity and localization of psoriasis were similar [8]. This might be the reason for the lack of therapeutic action against pruritus by administering oral anti-histamines in psoriatic patients. In addition, the increased dermal vasculature and angiogenesis in psoriasis also attract inflammatory cytokines that may further activate the mechanism of itch.

In studies, which correlate the increased level of cytokines to pruritus, it has been reported that interleukin (IL)-17-associated genes in scalp psoriasis are more active than other parts of the body involved with psoriasis [9]; elevated IL-23 gene transcription levels in psoriatic skin compared to healthy controls have been also reported [10]; in addition, there is an increased expression of Janus kinase (JAK) signal transducer and activation of transcription (STAT) pathway in psoriatic lesions [11]. IL-12 induces pruritus, presumably by inducing the propagation in Th1 differentiation and the further secretion of IL2 , known for its pruritogenic action [12, 13]. Likewise, tumor necrosis factor-a (TNF-a) upregulates the differentiation in Th1 resulting in itching psoriatic lesions [14]. The levels of TNF-a, IL-17, and IL-23 are presumably augmented also by phosphodiesterase-4 (PDE-4) via upregulating the cyclic adenosine monophosphate (cAMP) [15].

Therefore, the biologic agents and small molecules that inhibit the cytokines involved in the pathogenesis of the psoriatic skin, and specifically, scalp lesions may offer an effective treatment option in the management of scalp psoriasis, which, under a general agreement, belongs to the "difficult-to-treat" types of psoriasis. Even though the efficacy and safety of these drugs in the treatment of moderate-to-severe plaque psoriasis have already been established in several randomized controlled trials (RCT), few trials have investigated their therapeutic impact regarding scalp symptoms. Most clinical data are obtained by the sub-analysis of trials, in which scalp involvement is also assessed. In this review, we discuss the efficacy of the currently in-use biologic agents and small molecules, based on the evidence available in the scientific literature.

\section{Methods}

Search strategy included researching the databases of PubMed, Cochrane Library, and EMBASE for RCTs reporting the efficacy of biologics and small molecules in the treatment of scalp psoriasis.
In the current review, we included analysis and sub-analysis mainly of RCTs, investigating biological treatments and small molecules, currently approved by the FDA and EMA for the treatment of plaque psoriasis. The assessment of the psoriasis severity in the included RCTs was performed by applying the Psoriasis Area and Severity Index (PASI) for head and neck, the Psoriasis Scalp Severity Index (PSSI), the Scalp Physician Global Assessment (ScPGA), and the Scalp-Specific Investigator Global Assessment scale (ssIGA). Sub-analysis of RCTs reporting specific results for scalp psoriasis efficacy was included.

The effectiveness of the treatments in reducing scalp pruritus was described in the current review, while when the pruritus assessment in the RCTs was not performed specifically for the scalp, the results were denoted separately. No specific eligibility criteria concerning assessing of reduction of scalp pruritus were applied, due to lack of ubiquitously accepted measurement method.

\section{Biologic Agents}

\section{TNF-a Inhibitors}

This group is comprised of 4 agents: etanercept, infliximab, adalimumab, and certolizumab pegol.

\section{Etanercept}

Etanercept is a fusion protein that inhibits TNF-a, by competing the TNF-a binding with its receptors 1 and 2 . Primarily administered for psoriatic arthritis, it was approved by the FDA for the systemic treatment of adult plaque psoriasis in 2004, and later in 2016, the usage was expanded in pediatric patients suffering from the same disease.

In one RCT, 124 adult patients with plaque psoriasis and significant scalp symptoms (PASI scores of 10 or higher and $30 \%$ or higher of the scalp surface area affected, with PSSI scores of 15 or higher) were recruited [16]. Patients received etanercept $50 \mathrm{mg}$ twice weekly (BIW) for 12 weeks, followed by etanercept $50 \mathrm{mg}$ once weekly (QW) and placebo QW (Group A, $n=62$ ) or placebo BIW for 12 weeks, followed by etanercept $50 \mathrm{mg}$ BIW for 12 weeks (Group B, $n=62$ ). In week 12, mean PSSI improvement was $86.8 \% \pm 18 \%$ in group A and $20.4 \% \pm 39.3 \%$ in group B $(p<0.0001)$. Furthermore, 51 of $59(86 \%)$ group A patients and 7 of 61 (11\%) group B patients achieved PSSI75 (PSSI score decreased 75\%; $p<0.0001$ ) at week 12 . The degree and start-to-respond time of scalp psoriasis to etanercept were the same as in psoriasis in other body sites. When placebo-group patients were shifted from placebo to etanercept from week 12 and after, their response was similar to that of group A patients. At week 24, mean PSSI improvement was $90.6 \% \pm 13.1 \%$ in group $\mathrm{A}$ and $79.1 \%$ in group $\mathrm{B}$, demonstrating no significant difference. 
One sub-analysis of the above study, evaluating the impact of etanercept in the quality of life (QoL) of patients revealed that group A patients had significantly better results for all outcomes, including scalp pruritus, scalp pain, Dermatology Life Quality Index (DLQI), emotional distress or depression, and treatment satisfaction versus placebo [17]. Regarding group B, similar improvements were found after switching from placebo to etanercept following week 12 .

In another study with 250 patients receiving etanercept, placebo, and apremilast, a sustained decrease in pruritus Visual Analogue Scale (VAS) score (range 0-100 $\mathrm{mm}$ ) was observed in the etanercept and apremilast group of patients [18]. Beginning from the second week of drug administration, the improvement in pruritus was greater in the etanercept group $(-36.4 \mathrm{~mm}$ in VAS, $p<$ $0.001)$ followed by the apremilast group $(-35.6 \mathrm{~mm}$ in VAS, $p<00.026)$ versus the placebo group $(-22.5$ in VAS). At week 16, a decrease in VAS score more or equal to $20 \%$ was achieved in the $83.1 \%$ of the etanercept and the $79.5 \%$ of the apremilast group of patients, while the response of the placebo group was limited to 53.6\%. However, the design of the aforementioned study did not evaluate the improvement of pruritus in different anatomical regions.

\section{Infliximab}

Infliximab is a mouse/human chimeric antibody that specifically binds TNF-a, preventing the interaction of this ligand with its receptors. In 2006, infliximab was approved for the treatment of moderate/severe adult plaque psoriasis.

An analysis of infliximab effectiveness in scalp psoriasis in Japanese clinical trials included one RCT $(n=50)$ and one open-label trial $(n=64)$ [19-22]. Disease severity in the 4 body areas (head/neck, trunk, upper, and lower extremities) was calculated using PASI score separately for each area. The average improvement for the head area was $75.7 \%$ in the final assessment (week 62 in the RCT, week 46 in the open-label trial); $50.0 \%$ of patients achieved a $90 \%$ improvement in PASI score for the head, and $40.9 \%$ of patients exhibited $100 \%$ improvement in the final assessment.

In a retrospective, comparative study of 145 patients from our tertiary psoriasis referral center for the treatment of scalp psoriasis, in 35 patients infliximab was administered [23]. At week 4, the infliximab group achieved a better mean decrease in PSSI, with $74 \%$ of the patients achieving PSSI75. At week $48,70 \%$ of the patients were still maintaining the same response.

Scalp Psoriasis and Biologic Agents: A Review
The effectiveness of infliximab on different body regions was examined by analyzing data from 3 large-scale trials (EXPRESS, EXPRESS II, and SPIRIT) with a total of 1,462 patients [22]. After 10 weeks of treatment, the PASI response showed no difference among the 4 sites studied (head-neck, upper limbs, trunk, and lower limbs). At week 10, the results for the head-neck area are the following: $85.5 \%, 84.8 \%$, and $79 \%$ of patients achieved PASI 75 , while $68.9 \%, 73.1 \%$, and $66.6 \%$ of patients achieved PASI90 in the 3 trials, respectively. Overall, the infliximab showed good treatment efficacy for scalp psoriasis.

\section{Adalimumab}

Adalimumab is the first fully human monoclonal antibody that inhibits TNF-a. In detail, adalimumab is also bound to the TNF-a and prevents the interaction with the TNF receptors. In 2008, it was approved as a treatment for moderate-to-severe plaque psoriasis in adults, while in 2015 it was approved for pediatric usage.

Post hoc analysis of BELIEVE, a large phase III RCT, provided some evidence on the effectiveness of adalimumab in scalp psoriasis [24]. Of the 730 patients enrolled, $663(91.3 \%)$ and $433(60.1 \%)$ had psoriasis of the scalp or both nail and scalp, respectively. Patients received adalimumab $80 \mathrm{mg}$ at week $0,40 \mathrm{mg}$ at week 1 , and then adalimumab $40 \mathrm{mg}$ every other week until week 15 . The mean baseline PSSI score was 16.3. At week 16, the proportion of patients who achieved PSSI75 response was similar between patients with (68.2\%) and without (63.5\%) nail involvement. Patients with scalp psoriasis reported improvements in DLQI throughout the study, and the mean reduction in the PSSI score was $77.2 \%$ at week 16 .

In a case series, 13 patients with moderate-to-severe psoriasis presenting scalp lesions, who had an inadequate therapeutic response to etanercept, were enrolled [25]. The adalimumab was administered, and clinical response was evaluated at weeks 12 and 24 . Adalimumab failed to demonstrate a better effectiveness over etanercept in the treatment of scalp psoriasis, as there was no significant therapeutic difference between the 2 biologics.

In contrast, the effectiveness of adalimumab over 12 months in 404 patients with scalp psoriasis was evaluated in a multicenter prospective routine clinical setting [26]. The mean baseline PSSI score was 26.8 . The $93.8 \%$ of the patients achieved good clinical response upon treatment with adalimumab after 12 months, with $66.7 \%$ of them achieving complete remission of local symptoms. There was also a marked improvement in QoL index.

In another 16-week RCT in 271 adults with plaque psoriasis designed to evaluate the efficacy and the safety

Skin Appendage Disord 2021;7:439-448 441 
of adalimumab in the disease, a significant reduction in pruritus was revealed [27]. Pruritus was evaluated by applying the Psoriasis-Related Pruritus Assessment scale, a 10-point VAS. At week 16, there was a significant decrease of pruritus in all areas including scalp in the adalimumab group of patients. The score of reduction is 5.0 points in the treatment group versus a 1.8 points decrease in the placebo control group $(p<0.001)$. The assessment was performed without taking into consideration the different anatomical sites.

\section{Certolizumab Pegol}

Certolizumab pegol is a PEGylated Fab portion of a humanized monoclonal TNF-a antibody, approved for the treatment of moderate/severe plaque psoriasis in adults, since 2018. The PEGylation of the TNF-a antibody is a process of both covalent and noncovalent attachment of polyethylene glycol polymer chains onto the antibody. This process reduces the immunogenicity and antigenicity of the drug, and increases its size rendering the drug unable to penetrate the placenta or be transferred in the milk of breast-feeding women [28].

The efficacy of certolizumab pegol in scalp psoriasis, as well as in other difficult-to-treat areas, has not been described in the literature yet. However, results from 2 double-blinded, placebo RCTs in 559 and 227 patients (CIMPACT, CIMPASI-1, and CIMPASI-2) demonstrated a response rate of PASI75 in $>90 \%$ of them, after 16 weeks of treatment with certolizumab pegol [29]. However, effectiveness in different body areas is not specified. Data about the effect of the drug in reducing pruritus are also lacking to this day.

\section{IL-12 and IL-23 Inhibitors}

The biologics acting in this axe, namely, ustekinumab (IL-12 and IL-23), tildrakizumab (IL-23), guselkumab (IL-23), and risankizumab (IL-23), are included.

\section{Ustekinumab}

Ustekinumab is a fully human monoclonal antibody that directly inhibits IL-12 and IL-23 by binding their shared p40 subunit. It was approved for adult use in patients suffering from plaque psoriasis in 2009, and in 2020, it received the FDA approval for pediatric usage.

No published RCTs have investigated ustekinumab specifically for the treatment of scalp psoriasis. Case reports described rapid (8 weeks) scalp symptoms response and itching remission, as well as a significant improvement in the QoL, following ustekinumab treatment $[30,31]$. A retrospective, comparative study of
145 patients from our tertiary psoriasis referral center compares 4 biologic agents (infliximab, etanercept, adalimumab, and ustekinumab) in the treatment of scalp psoriasis symptoms [23]. At week 4, the 41-ustekinumab patients achieved a $62 \%$ mean decrease in the PSSI, whereas the decrease in the PSSI score at week 48 was $94.9 \%$. Noteworthy data about the effect of ustekinumab in pruritus lack in the literature and mainly refer to isolated cases.

\section{Tildrakizumab}

Tildrakizumab is a humanized IgG antibody against the p19 subunit of IL-23. As a result, this agent acts only against the IL-23. The FDA approval for moderate-tosevere plaque psoriasis arrived in 2018.

Although RCTs for tildrakizumab demonstrated the efficacy of the drug in terms of PASI and PGA scores (SURFACE 1 and 2), assessments regarding the effectiveness of the treatment in the scalp or in specific anatomical - difficult to treat - positions are still lacking $[32,33]$.

\section{Guselkumab}

Guselkumab is a fully human IL-23p19 monoclonal antibody, approved for the treatment of moderate-to-severe plaque psoriasis by the FDA in 2017.

Results from the phase III, RCT, VOYAGE study - including $78 \%$ of patients with moderate/severe scalp psoriasis at baseline - showed that the proportion of patients in the guselkumab group achieving scalp-specific IGA 0/1 was significantly higher compared with placebo (83.4 vs. $14.5 \%, p<0.001)$ at week 16 . Moreover, significantly better responses to guselkumab versus adalimumab were observed at weeks 24 ( 84.5 vs. 69.2$)$ and 48 (78.3 vs. 60.5 ) (both $p<00.001$ ) [34].

A pooled, post hoc analysis of the 2 pivotal phase III RCTs comparing the efficacy in specific body regions of guselkumab (VOYAGE 1 and VOYAGE 2 trials) versus adalimumab and placebo showed that of 1,829 randomized patients, the $86.2 \%$ had psoriasis of the scalp, while $82.7 \%$ had a score of 2 or higher on the ss-IGA score and were included in the analysis [35]. Guselkumab appeared superior to placebo based on the proportion of patients achieving an ss-IGA score of 0 or 1 (81.8 vs. $12.4 \%)$ at week 16 and to adalimumab ( 85.0 vs. $68.5 \%$ ) at week 24 (both $p<0.001$ ). More importantly at week $24,69.9 \%$ of patients treated with guselkumab versus $56.3 \%$ treated with adalimumab group achieved an ss-IGA score of 0 (all $p<0.001)$. In conclusion, compared to adalimumab, guselkumab was associated with a significantly better improvement in scalp psoriasis. 
Risankizumab

Risankizumab is a humanized IgG1 monoclonal IL23 p19 antibody. FDA approved risankizumab for the treatment of moderate-to-severe plaque psoriasis for adults in 2019.

In one randomized, head-to-head, dose-ranging phase 2 trial, 166 patients randomized in 1:1:1:1 ratio received a single dose of risankizumab 18, 90, $180 \mathrm{mg}$, or ustekinumab $45 \mathrm{mg}$ (with bodyweight $<90 \mathrm{~kg}$ ) and $90 \mathrm{mg}$ (bodyweight $\geq 90 \mathrm{~kg}$ ) at weeks 0,4 , and 16 , respectively [36]. The group of patients receiving $90 \mathrm{mg}$ of risankizumab achieved a $90 \%$ mean PSSI reduction at week 12, while those receiving $180 \mathrm{mg}$ achieved a reduction of $94 \%$ of PSSI for the same period of treatment. The control group of patients receiving ustekinumab achieved an $82 \%$ reduction of PSSI for the same treatment period, but the results were not sustained over time.

\section{IL-17 Inhibitors}

The biologics acting in this axe are secukinumab, ixekizumab, and brodalumab.

\section{Secukinumab}

Secukinumab is a fully human monoclonal antibody that selectively targets IL-17A. Approval from the FDA for its use in the treatment of adults with plaque psoriasis was granted in 2015.

A phase-3b RCT "SCALP" analyzed the effects of secukinumab in moderate-to-severe scalp psoriasis [37]. Adult patients diagnosed with moderate-to-severe scalp psoriasis of $\geq 6$-month duration, inadequately controlled by topical treatments or systemic therapies, were eligible to participate in the trial. It is worth noting that patients with or without plaque psoriasis elsewhere on the body were equally eligible.

Moderate-to-severe scalp psoriasis was defined as a PSSI score of $\geq 12$, with $\geq 30 \%$ of the scalp surface area affected and an Investigator's Global Assessment modified 2011 score of $\geq 3$. The objectives of the study included determining the number of patients that improved by $90 \%$ in the PSSI, and achieved an IGA score of 0 (clear) or 1 (almost clear) at week 12. Additionally, the following patient-reported symptoms were evaluated: scalp pain, itching, and scaling at baseline, and then at weeks 1, 2, 3, 4, 8, and 12. A total of 102 patients were randomized 1:1 to receiving either secukinumab $300 \mathrm{mg}$ or placebo at baseline. At 12 weeks, patients on placebo who did not achieve a PSSI90 were switched to secukinumab $300 \mathrm{mg}$ until week 24. Patients on placebo that achieved PSSI90 continued on placebo until the end of the study. All groups

Scalp Psoriasis and Biologic Agents: A Review remained blinded during the course of the study. The patients of both groups were balanced according to baseline characteristics such as age, gender, BMI, and baseline PSSI and PASI scores. At week 12, PSSI90 was achieved in $52.9 \%$ of the patients in the secukinumab group versus $2 \%$ in the placebo group. At week 24, the percentage of treated patients that achieved PSSI90 reached $58.8 \%$. The IGA scalp score of $0 / 1$ was achieved by $56.9 \%$ in the secukinumab group versus $5.9 \%$ in the placebo group. Additionally, at 12 weeks, $35 \%$ of the patients in the secukinumab group achieved a PSSI 100 compared to $0 \%$ in the placebo group, and at week 24 , the rate of patients with PSSI100 reached 47.2\% ( $p<0.001)$. Overall, QoL improvement was significantly higher in the secukinumab-treated group compared to the placebo group $(p<$ $0.001)$. Patients treated with secukinumab also recorded a statistically significant greater mean improvement in scalp itching, scaling, and pain compared to placebo group.

Furthermore, a pooled analysis of 4 phase III RCTs (ERASURE, FIXTURE, FEATURE, and JUNCTURE) assessed the efficacy of secukinumab for head and neck psoriasis in patients with moderate-to-severe disease [38]. This sub-analysis did not assess scalp psoriasis separately. At 52 weeks, head and neck PASI90 and 100 responses were achieved by 76.0 and $68.7 \%$ of the patients receiving secukinumab $300 \mathrm{mg}$, respectively. At week 52, wholebody PASI90 and 100 responses were achieved by 68.1 and $40.8 \%$ of the patients receiving secukinumab $300 \mathrm{mg}$, respectively.

Phase III RCT ERASURE and FIXTURE reported a significant decrease in pruritus. Assessment was performed using the patient-reported Psoriasis Symptom Diary (PSD) [38]. Analysis of the data collected with the usage of the aforementioned $0-10$ scale showed a decrease of at least 2.2 points in $83 \%$ of the patients. However, position-correlated assessment is absent.

\section{Ixekizumab}

Ixekizumab is a humanized IL-17A monoclonal antibody. It was approved by the FDA in 2016 for the treatment of plaque psoriasis in adults.

A phase II RCT demonstrated improvement in scalp psoriasis following treatment with ixekizumab [39]. Patients $(n=142)$ were randomized to receive placebo, 10 , 25,75 , or $150 \mathrm{mg}$ of ixekizumab at weeks $0,2,4,8,12$, and $16 ; 105$ patients $(74.0 \%)$ had mean scalp psoriasis PSSI at $18.7( \pm 12.8)$ at baseline. The trial had 2 periods: an initial 20 -week RCT period and a subsequent 48-week open-label extension period. Significant improvement in terms 
of PSSI reduction was reported as early as week 1 in ixekizumab $150 \mathrm{mg}$ group with a decrease of 8.7 points of baseline PSSI (37.4\%, $p=0.003)$ versus placebo $(-2.4)(10.9 \%)$. At week 4, the ixekizumab patients presented a PSSI reduction of 12.3 points $(66.6 \%, p=0.03)$ in the $25 \mathrm{mg}$ group, $9.7(77.4 \%, p=0.01)$ in the $75 \mathrm{mg}$ group, and 17.9 $(86.2 \%, p<0.001)$ in the $150 \mathrm{mg}$ group, compared to placebo with $6.4(26.9 \%)$. At week 20, patients with scalp psoriasis in the $25 \mathrm{mg}, 75 \mathrm{mg}$, and $150 \mathrm{mg}$ groups had significant mean changes from baseline PSSI, with a reduction of $16.3(75.3 \%, p=0.001), 11.6(83.7 \%, p=0.001)$, and $18.2(82.2 \%, p<0.001)$, respectively, compared to 6.0 (18.8\%) in the placebo group. At week 48 , a total of $81.3 \%$ of patients with scalp psoriasis receiving $120 \mathrm{mg}$ ixekizumab experienced complete resolution of lesions.

In a pooled subgroup analysis of 3 phase III RCTs (UNCOVER 1, 2, 3), patients with moderate-to-severe psoriasis with baseline scalp involvement were assessed [40]. Approximately half of the scalp psoriasis patients in each treatment group met criteria for more severe scalp psoriasis (PSSI $\geq 15$ and $\geq 30 \%$ scalp involvement). At week 12, PSSI75, 90, and 100 were achieved in 89.9, 81.7, and $74.6 \%$ of the patients treated with ixekizumab, respectively. These outcomes remained stable through week 60 of the maintenance period in patients who continued receiving ixekizumab every 4 weeks (Q4W). Patients who received ixekizumab every 2 weeks (Q2W) reached an average 93\% improvement in PSSI versus 70\% in the etanercept group $(p<0.001)$ and $3.7 \%$ in the placebo group $(p<0.001)$. The subgroup of patients with more severe scalp involvement $(n=1,767)$ at baseline (PSSI $\geq 15$ and $\geq 30 \%$ ) had comparable improvement in scalp psoriasis compared to those patients with moderate scalp disease. In detail, $90.9,82.2$, and $69.8 \%$ of patients with more severe disease achieved PSSI75, 90, and 100, respectively, after 12 weeks of drug administration. The median times for patients achieving PSSI75 and 90 were comparable between patients with any baseline scalp psoriasis and more severe scalp psoriasis (2.1 and 4.1 weeks, respectively).

In IXORA-S, a multicenter, double-blinded, parallelgroup trial, 3 different ixekizumab dosing groups of patients presenting scalp psoriasis with mean PSSI of 21.3, 19.4 , and 19.9, respectively, were assessed for efficacy after 52 weeks of treatment [41]. The 3 groups received ixekizumab $80 \mathrm{mg}$ Q2W, Q4W, and Q4W with adjustment to Q2W when specified criteria were met, until the end point at 52 weeks. Total PSSI clearance was achieved in $76.9 \%$ of the patients treated with Q2W, in $70.3 \%$ with Q4W group, and in the $72.6 \%$ in the dose-adjustment group (Q4W/Q2W). In the same trial, a significant reduction of pruritus was demonstrated in $75 \%$ of all ixekizumab patients, but the assessment was not performed regarding the different anatomical parts.

\section{Brodalumab}

Brodalumab is a human anti-interleukin-17 receptor A (IL-17RA) monoclonal antibody approved by the FDA in 2017 for use in patients with moderate-to-severe plaque psoriasis. Brodalumab improved difficult-totreat scalp psoriasis, according to phase-3 AMAGINE-1 study [42]. Patients with scalp psoriasis $(n=222$ receiving brodalumab and $n=219$ receiving placebo), presenting PSSI score equal or greater than 15 , were enrolled. The patients were randomized and received brodalumab $210 \mathrm{mg}$ or placebo, during the 12 -week induction period. Mean percent improvement rates from baseline PSSI demonstrated a rate of reduction equal to $87.5 \%$ for brodalumab versus $7.1 \%(p<0.001)$ for placebo patients, as early as week 4 . Mean improvement from baseline to week 12 favored brodalumab over placebo $(p \geq 0.001)$, with the 89 and $63.4 \%$ of brodalumab patients, and the 9.4 and $3.2 \%$ placebo patients, achieving PSSI75 and PSSI90, respectively.

\section{Small Molecules}

Currently in this category, the only existing small molecule is apremilast.

\section{Apremilast}

Apremilast is an oral PDE-4 inhibitor, approved for the treatment of adult plaque psoriasis since 2014. Apremilast acts by increasing intracellular cAMP levels and thus regulating the production of pro-inflammatory and anti-inflammatory mediators.

A pooled analysis of 2 phase III RCTs, ESTEEM 1, and ESTEEM 2, reported the effects of apremilast in difficultto-treat nail and scalp psoriasis [43]. In total, 1,255 patients were randomized (2:1) to receive apremilast $30 \mathrm{mg}$ twice daily or placebo. At week 16, placebo patients switched to apremilast through week 32 , followed by a randomized withdrawal phase to week 52 . A priori efficacy analyses included patients with moderate-to-severe scalp (ScPGA score $\geq 3$ ) psoriasis at baseline. Patients who did not achieve a PASI75 (ESTEEM 1) or PASI50 (ESTEEM 2) response at week 32 were able to use topical agents and/or UVB therapy. At baseline, 66.7 and 65.5\% of patients had moderate-to-severe scalp psoriasis in ESTEEM 1 and 2, respectively. Significantly more patients receiving apremilast achieved an ScPGA score of 0 or 1 at 
week 16 (46.5 vs. $17.5 \%$ ESTEEM 1; 40.9 vs. $17.2 \%$ ESTEEM 2; $p \geq 0.0001$ for both) than those receiving placebo. Improvement in scalp involvement was maintained through week 32 among patients initially randomized to apremilast (37.4 and 32.4\% patients in ESTEEM 1 and ESTEEM 2, with ScPGA score of 0 or 1 ). Among patients initially randomized to placebo who switched to apremilast at week 16, a total of $71(43.6 \%)$ and $36(50.7 \%)$ patients in ESTEEM 1 and ESTEEM 2, respectively, achieved an improvement in ScPGA score of 0 or 1 at week 32 . Improvements were maintained over week 52 .

Furthermore, findings from a phase IIIb LIBERATE study assessing the efficacy of apremilast through 104 weeks in moderate-to-severe scalp psoriasis who continued on apremilast or switched from etanercept treatment are presented [44]. Two hundred and fifty patients were randomized to placebo, apremilast $30 \mathrm{mg}$ BID, or etanercept $50 \mathrm{mg}$ QW through week 16; thereafter, all patients continued or switched to apremilast through week 104 (extension phase). Skin, scalp, and nail psoriasis involvement at weeks 16, 52, and 104 were assessed, but the baseline characteristics of scalp psoriasis were not given. At weeks 52 and 104, ScPGA response of 0 (clear) or 1 (minimal) was achieved by $52.0-60.4$ and $50.0-59.2 \%$ of patients, respectively.

\section{Discussion}

Psoriasis of the scalp is common, it may occur with or without cutaneous involvement elsewhere, and it constitutes a difficult-to-treat manifestation of the disease. Researching the efficacy of existing biologic treatments in reducing scalp psoriasis lesions and minimizing pruritus was the main aim of the current review. The results indicate that the biologics used for the treatment of skin plaque psoriasis are also helpful in controlling the disease affecting the scalp.

In general, various types of clinical trials, sub-analyses, and post hoc analyses were published and included in the review, with different dimensions and severity of scalp psoriasis in the populations under examination. There were studies evaluating biologic treatment efficacy in patients with psoriasis affecting the scalp, in addition to other skin areas, or in patients affecting the scalp as the only type of involvement. The aforementioned study design differences render the comparison among the biologics difficult. What is more, heterogeneity in scoring measurements, timeline end points, and drug dosing adopted, along with disparity in the statistical methodology ap-

Scalp Psoriasis and Biologic Agents: A Review plied, were the major reasons for discrepancies among trials included in the present review.

Acknowledging that indirect comparisons between trials are not advisable, an effort was made to outline the efficacy of the biologic drugs under evaluation. The assessment of their efficacy was performed by dividing the response rate in accordance with timeline. Early response to treatment is measured in the first 8 weeks of drug administration, late response in 16 weeks, while drug survival was assessed for treatment efficacy after 48 weeks of drug administration (Table 1).

Early response efficacy demonstrates superiority of infliximab, with $74 \%$ of the patients achieving PSSI75 from week 4 of the administration of the drug. A head-to-head comparison between ixekizumab and infliximab demonstrates that in both groups, $>75 \%$ of the patients achieved PSSI75 at week 8. Patients who received ustekinumab demonstrated a mean PSSI decrease of $62 \%$ from the baseline, after 4 weeks of drug administration.

After 10 weeks of drug administration, approximately the $85.5 \%$ of infliximab patients presented a PASI75 in 3 RCTs for neck and scalp psoriasis, without, however, exhibiting details for the efficacy in scalp psoriasis individually. For the same period of treatment, ixekizumab patients present a mean PSSI decrease of almost $90 \%$.

More data are available for the treatment period between weeks 12 and 16. Ixekizumab proved its efficacy with $90.3,83.1$, and $75.6 \%$ of the patients achieving PSSI75, 90, and 100, respectively. Brodalumab follows closely with $89 \%$ of the patients achieving PSSI75 and 63.4\% PSSI90 after 12 weeks of treatment, and etanercept with $86 \%$ of the patients referring PSSI75 reduction for the same period.

Mean PSSI reduction of 94 and 90\% for ustekinumab and risankizumab patients, respectively, after 12 weeks of treatment is reported. Trials for adalimumab report a mean PSSI reduction of $77.2 \%$ after 16 weeks of treatment. After 16 weeks of treatment, the $81.8 \%$ of the guselkumab patients achieved ss-IGA equal to 0 or 1 , while 2 clinical trials for apremilast report ScPGA equal to 0 or 1 in 46.5 and $40.9 \%$ of patients, respectively. Complete treatment response within the first 16 weeks of drug administered, in terms of PSSI100, is reported in the case 2 biologics: $74.6 \%$ of the ixekizumab patients and $35 \%$ of the secukinumab one.

Long-term efficacy in the ixekizumab trials reports a $93 \%$ improvement on PSSI after 60 weeks, with $75.9 \%$ of them presenting total clearance (PSSI $=0$ ) after 52 weeks. The infliximab trials report a PASI75 response for $70 \%$ of the patients after 48 weeks, while secukinumab proved

Skin Appendage Disord 2021;7:439-448 445 
Table 1. Biological treatments response, divided in early ( $<8$ weeks), late ( $8-16$ weeks), and drug survival ( $>48$ weeks)

\begin{tabular}{|c|c|c|c|c|c|}
\hline Biologic pathway & Biologics & Early response & Late response & Drug survival & Reference \\
\hline \multirow[t]{6}{*}{ TNF-a inhibitors } & Etanercept & & $\begin{array}{l}86 \% \text { PSSI75 } \\
86.8 \% \text { mean PSSI reduction }\end{array}$ & & Bagel et al. [16] \\
\hline & Infliximab & & & $\begin{array}{l}75.7 \% \text { mean PASI } \\
\text { reduction }\end{array}$ & Torii et al. [21] \\
\hline & & $74 \%$ mean PASI reduction & & & Fotiadou et al. [23] \\
\hline & Adalimumab & & $77.2 \%$ PASI75 & & Thaci et al. [24] \\
\hline & & & & $66.7 \%$ PSSI100 & Khobzey et al. [26] \\
\hline & Certolizumab pegol & & & & $\begin{array}{l}\text { Not existing clinical } \\
\text { trials }\end{array}$ \\
\hline \multirow{3}{*}{$\begin{array}{l}\text { IL-12 and IL-23 } \\
\text { inhibitors }\end{array}$} & Guselkumab & & $83.4 \%$ IGA 0 or 1 & $78.3 \%$ IGA 0 or 1 & Blauvelt et al. [34] \\
\hline & & & $81.8 \%$ IGA 0 or 1 & $85 \%$ IGA 0 or 1 & Foley et al. [35] \\
\hline & Risankizumab & & $\begin{array}{l}\text { 90\% mean PSSI reduction } \\
\text { (dose: } 90 \mathrm{mg} \text { ) }\end{array}$ & & Papp et al. [36] \\
\hline \multirow[t]{3}{*}{ IL-17 inhibitors } & Secukinumab & & $\begin{array}{l}52.9 \% \text { PSSI90 } \\
56.9 \% \text { IGA } 0 \text { or } 1 \\
35 \% \text { PSSI } 100\end{array}$ & & Bagel et al. [37] \\
\hline & & & & $\begin{array}{l}\text { 76\% PASI90* } \\
68.7 \% \text { PASI100* }\end{array}$ & Kircik et al. [38] \\
\hline & Brodalumab & & $\begin{array}{l}89 \% \text { PSSI75 } \\
63.4 \% \text { PSSI } 90\end{array}$ & & Elewski et al. [42] \\
\hline \multirow[t]{2}{*}{ Small molecules } & Apremilast & & $\begin{array}{l}46.5 \% \text { ScPGA } 0 \text { or } 1 \\
40.9 \% \text { ScPGA } 0 \text { or } 1\end{array}$ & $\begin{array}{l}\text { 83.3\% ScPGA } 0 \text { or } 1 \\
62.5 \% \text { ScPGA } 0 \text { or } 1\end{array}$ & Rich et al. [43] \\
\hline & & & & $52-60.4 \%$ ScPGA 0 or & Reich et al. [44] \\
\hline
\end{tabular}

PSSI, Psoriasis Scalp Severity Index; PASI, Psoriasis Area and Severity Index; ScPGA, Scalp Physician Global Assessment; IL, interleukin. Blank fields indicate data that are not available or not provided by the studies. * PASI calculated for head and neck.

more effective with 76 and $68.7 \%$ achieving PASI90 and PASI100, after 52 weeks, respectively. After 48 weeks of treatment, $66.7 \%$ of the adalimumab patients achieved PSSI100, while 52 and $50 \%$ of the apremilast patients present ScPGA 0/1 after 52 and 104 weeks, respectively.
Regarding improvement in scalp pruritus, the diversity in measurement methods renders comparisons among biologics not possible to accomplish. It is a prerequisite that uniform measurement methods be applied in all RCTs, in order to facilitate comparisons among biologics. Finally, while early response treatments are pref-
Papadimitriou/Bakirtzi/Katoulis/ Ioannides 
erable in some cases of severe scalp psoriasis, in the majority of scalp psoriatic patients long-term efficacy and drug safety could be the major criteria for choosing the appropriate treatment.

Scalp-located psoriasis presents a challenging pathology for the clinician to treat and a real burden for the patients' everyday life. Although the progress in biologics has advanced notably in terms of controlling the skin psoriatic plaques and the symptoms that follow, the localization of the disease on the scalp still poses difficulties in the rapid and effective skin clearance. Impartial data for the attenuation of skin symptoms - such as pruritus - are extremely rare and the existing ones are assessed with the VAS, which does not offer a meticulous appraisal of pruritus in the everyday life. Even though the impact of scalp pruritus and scalp scaling in the patients' quality of life is often well reflected in the DLQI questionnaire, clinicians should also assess the potential burden themselves, therefore protecting them from further deterioration of their life quality.

\section{Conflict of Interest Statement}

The authors have no conflicts of interest to declare.

\section{Funding Sources}

The authors have no funding sources to declare.

\section{Author Contributions}

Ilias Papadimitriou is the writer of the main text and involved in data collection and analysis. Katerina Bakirtzi involved in drafting the work and data collecting. Alexander Katoulis involved in drafting the work and data collecting. Dimitrios Ioannides involved in conception and design of the work, proofreading, revising, and final approval.

\section{References}

1 Chan CS, Van Voorhees AS, Lebwohl MG, Korman NJ, Young M, Bebo BF Jr, et al. Treatment of severe scalp psoriasis: from the Medical Board of the National Psoriasis Foundation. J Am Acad Dermatol. 2009;60(6):96271.

2 Farber EM, Nall L. Natural history and treatment of scalp psoriasis. Cutis. 1992;49(6):396.

3 van de Kerkhof PC, Franssen ME. Psoriasis of the scalp. Diagnosis and management. Am J Clin Dermatol. 2001;2(3):159-65.

4 Mason AR, Mason JM, Cork MJ, Hancock H, Dooley G. Topical treatments for chronic plaque psoriasis of the scalp: a systematic review. Br J Dermatol. 2013;169(3):519-27.

5 Rouzaud M, Sevrain M, Villani AP, Barnetche T, Paul C, Richard MA, et al. Is there a psoriasis skin phenotype associated with psoriatic arthritis? Systematic literature review. J Eur Acad Dermatol Venereol. 2014;28(Suppl 5):17-26.

6 Wilson FC, Icen M, Crowson CS, McEvoy MT, Gabriel SE, Kremers HM. Incidence and clinical predictors of psoriatic arthritis in patients with psoriasis: a population-based study. Arthritis Rheum. 2009;61(2):233-9.

7 Leon A, Rosen JD, Hashimoto T, Fostini AC, Paus R, Yosipovitch G. Itching for an answer: a review of potential mechanisms of scalp itch in psoriasis. Exp Dermatol. 2019;28(12): 1397-404.

8 Wiśnicka B, Szepietowski J, Reich A, Orda A. Histamine, substance $\mathrm{P}$ and calcitonin generelated peptide plasma concentration and pruritus in patients suffering from psoriasis. Dermatol Psychosom. 2004;5(2):73-8.
9 Brembilla NC, Senra L, Boehncke WH. The IL-17 family of cytokines in psoriasis: IL-17A and beyond. Front Immunol. 2018;9:1682.

10 Nattkemper LA, Tey HL, Valdes-Rodriguez R, Lee H, Mollanazar NK, Albornoz C, et al. The genetics of chronic itch: gene expression in the skin of patients with atopic dermatitis and psoriasis with severe itch. Invest Dermatol. 2018;138(6):1311-7.

11 Andrés RM, Hald A, Johansen C, Kragballe K, Iversen L. Studies of Jak/STAT3 expression and signalling in psoriasis identifies STAT3Ser727 phosphorylation as a modulator of transcriptional activity. Exp Dermatol. 2013; 22(5):323-8.

12 Leonardi CL, Kimball AB, Papp KA, Yeilding N, Guzzo C, Wang Y, et al. Efficacy and safety of ustekinumab, a human interleukin-12/23 monoclonal antibody, in patients with psoriasis: 76-week results from a randomised, double-blind, placebo-controlled trial (PHOENIX 1). Lancet. 2008;371(9625):1665-74.

13 Nakamura M, Toyoda M, Morohashi M. Pruritogenic mediators in psoriasis vulgaris: comparative evaluation of itch-associated cutaneous factors. Br J Dermatol. 2003;149(4): 718-30.

14 Sola-Ortigosa J, Sánchez-Regaña M, UmbertMillet P. Efficacy of adalimumab in the treatment of psoriasis: a retrospective study of 15 patients in daily practice. J Dermatolog Treat. 2012;23(3):203-7.

15 Sobell JM, Foley P, Toth D, Mrowietz U, Girolomoni G, Goncalves J, et al. Effects of apremilast on pruritus and skin discomfort/pain correlate with improvements in quality of life in patients with moderate to severe plaque psoriasis. Acta Derm Venereol. 2016;96(4): 514-20.

16 Bagel J, Lynde C, Tyring S, Kricorian G, Shi Y, Klekotka P. Moderate to severe plaque psoriasis with scalp involvement: a randomized, double-blind, placebo-controlled study of etanercept. J Am Acad Dermatol. 2012;67(1): 86-92.

17 Tyring S, Bagel J, Lynde C, Klekotka P, Thompson E, Gandra S, et al. Patient-reported outcomes in moderate-to-severe plaque psoriasis with scalp involvement: results from a randomized, double-blind, placebo-controlled study of etanercept. J Eur Acad Dermatol Venereol. 2013;27(1):125-8.

18 Green L, Thaci D, Zhang Z, Goncalves J, Nograles K, Nikkels A. Effect of apremilast and etanercept on pruritus and health-related quality of life in patients with moderate to severe plaque psoriasis: results from the LIBERATE study. J Am Acad Dermatol. 2016;74(5): AB245.

19 Torii H, Sato N, Yoshinari T, Nakagawa H, Investigators JIS. Dramatic impact of a psoriasis area and severity index 90 response on the quality of life in patients with psoriasis: an analysis of Japanese clinical trials of infliximab. J Dermatol. 2012;39(3):2539.

20 Torii $\mathrm{H}$, Nakagawa $\mathrm{H}$. Japanese Infliximab Study i. Infliximab monotherapy in Japanese patients with moderate-to-severe plaque psoriasis and psoriatic arthritis. A randomized, double-blind, placebo-controlled multicenter trial. J Dermatol. 2010;59(1):40-9. 
21 Torii H, Nakagawa H, Investigators JIS. Longterm study of infliximab in Japanese patients with plaque psoriasis, psoriatic arthritis, pustular psoriasis and psoriatic erythroderma. J Dermatol. 2011;38(4):321-34.

22 Menter A, Reich K, Li S, Guzzo C. Consistency of infliximab response in different body regions for treatment of moderate to severe psoriasis: results from controlled clinical trials. J Am Acad Dermatol. 2008;58(2):Ab120.

23 Fotiadou C, Lazaridou E, Sotiriou E, Kyrgidis A, Apalla Z, Ioannides D. Scalp psoriasis and biologic agents: a retrospective, comparative study from a tertiary psoriasis referral centre. J Eur Acad Dermatol Venereol. 2016;30(12): 2091-6.

24 Thaci D, Unnebrink K, Sundaram M, Sood S, Yamaguchi Y. Adalimumab for the treatment of moderate to severe psoriasis: subanalysis of effects on scalp and nails in the BELIEVE study. J Eur Acad Dermatol Venereol. 2015; 29(2):353-60.

25 Wang T-S, Tsai T-F. Safety and effectiveness of adalimumab in patients with moderate-tosevere psoriasis who had inadequate therapeutic response to prior etanercept. Dermatologica Sinica. 2013;31(1):11-8.

26 Khobzey K, Liskova I, Szegedi A, Pavlovsky L, Lunder T, Kingo K, et al. Effectiveness of adalimumab in the treatment of scalp and nail affection in patients with moderate to severe plaque psoriasis in routine clinical practice. Acta Dermatovenerol Alp Pannonica Adriat. 2017;26(1):11-4.

27 Revicki D, Willian MK, Saurat JH, Papp KA, Ortonne JP, Sexton C, et al. Impact of adalimumab treatment on health-related quality of life and other patient-reported outcomes: results from a 16-week randomized controlled trial in patients with moderate to severe plaque psoriasis. Br J Dermatol. 2008;158(3): 549-57.

28 Pasut G. Pegylation of biological molecules and potential benefits: pharmacological properties of certolizumab pegol. BioDrugs. 2014; 28(S1):15-23.
29 Gottlieb AB, Blauvelt A, Thaçi D, Leonardi CL, Poulin Y, Drew J, et al. Certolizumab pegol for the treatment of chronic plaque psoriasis: results through 48 weeks from 2 phase 3 , multicenter, randomized, double-blinded, placebo-controlled studies (CIMPASI-1 and CIMPASI-2). J Am Acad Dermatol. 2018; 79(2):302-14.e6

30 Di Cesare A, Fargnoli MC, Peris K. Rapid response of scalp psoriasis to ustekinumab. Eur J Dermatol. 2012;21(6):993-4.

31 Papadavid E, Ferra D, Koumaki D, Dalamaga M, Stamou C, Theodoropoulos K, et al. Ustekinumab induces fast response and maintenance of very severe refractory scalp psoriasis: results in two Greek patients from the psoriasis hospital-based clinic. Dermatology. 2014;228(2):107-11.

32 Reich K, Warren RB, Iversen L, Puig L, PauCharles I, Igarashi A, et al. Long-term efficacy and safety of tildrakizumab for moderate-tosevere psoriasis: pooled analyses of two randomized phase III clinical trials (reSURFACE 1 and reSURFACE 2) through 148 weeks. $\mathrm{Br}$ J Dermatol. 2020;182(3):605-17.

33 Reich K, Papp KA, Blauvelt A, Tyring SK, Sinclair $\mathrm{R}$, Thaçi $\mathrm{D}$, et al. Tildrakizumab versus placebo or etanercept for chronic plaque psoriasis (reSURFACE 1 and reSURFACE 2): results from two randomised controlled, phase 3 trials. Lancet. 2017;390(10091):276-88.

34 Blauvelt A, Papp KA, Griffiths CEM, Randazzo B, Wasfi Y, Shen Y-K, et al. Efficacy and safety of guselkumab, an anti-interleukin-23 monoclonal antibody, compared with adalimumab for the continuous treatment of patients with moderate to severe psoriasis: results from the phase III, double-blinded, placebo- and active comparator-controlled VOYAGE 1 trial. J Am Acad Dermatol. 2017;76(3):405-17.

35 Foley P, Gordon K, Griffiths CEM, Wasfi Y, Randazzo B, Song M, et al. Efficacy of guselkumab compared with adalimumab and placebo for psoriasis in specific body regions: a secondary analysis of 2 randomized clinical trials. JAMA Dermatol. 2018;154(6):676-83.

36 Papp KA, Blauvelt A, Bukhalo M, Gooderham M, Krueger JG, Lacour JP, et al. Risankizumab versus ustekinumab for moderate-to-severe plaque psoriasis. N Engl J Med. 2017;376(16): 1551-60.
37 Bagel J, Duffin KC, Moore A, Ferris LK, Siu K, Steadman J, et al. The effect of secukinumab on moderate-to-severe scalp psoriasis: results of a 24-week, randomized, double-blind, placebo-controlled phase 3b study. J Am Acad Dermatol. 2017;77(4):667-74.

38 Kircik L, Fowler J, Weiss J, Meng X, Guana A, Nyirady J. Efficacy of secukinumab for moderate-to-severe head and neck psoriasis over 52 weeks: pooled analysis of four phase 3 studies. Dermatol Ther. 2016;6(4):627-38.

39 Langley RG, Rich P, Menter A, Krueger G, Goldblum O, Dutronc Y, et al. Improvement of scalp and nail lesions with ixekizumab in a phase 2 trial in patients with chronic plaque psoriasis. J Eur Acad Dermatol Venereol. 2015;29(9):1763-70

40 Reich K, Leonardi C, Lebwohl M, Kerdel F, Okubo Y, Romiti R, et al. Sustained response with ixekizumab treatment of moderate-tosevere psoriasis with scalp involvement: results from three phase 3 trials (UNCOVER-1, UNCOVER-2, UNCOVER-3). J Dermatolog Treat. 2017;28(4):282-7.

41 Langley R, Papp K, Gooderham M, Zhang L, Mallinckrodt C, Agada N, et al. Efficacy and safety of continuous every-2-week dosing of ixekizumab over 52 weeks in patients with moderate-to-severe plaque psoriasis in a randomized phase III trial (IXORA-P). Br J Dermatol. 2018;178(6):1315-23.

42 Elewski B, Rich P, Lain E, Soung J, Lewitt GM, Jacobson A. Efficacy of brodalumab in the treatment of scalp and nail psoriasis: results from three phase 3 trials. J Dermatolog Treat. 2020:1-5.

43 Rich P, Gooderham M, Bachelez H, Goncalves J, Day RM, Chen R, et al. Apremilast, an oral phosphodiesterase 4 inhibitor, in patients with difficult-to-treat nail and scalp psoriasis: results of 2 phase III randomized, controlled trials (ESTEEM 1 and ESTEEM 2). J Am Acad Dermatol. 2016;74(1):134-42.

44 Reich K, Gooderham M, Bewley A, Green L, Soung J, Petric R, et al. Safety and efficacy of apremilast through 104 weeks in patients with moderate to severe psoriasis who continued on apremilast or switched from etanercept treatment: findings from the LIBERATE study. J Eur Acad Dermatol Venereol. 2018; 32(3):397-402. 